\title{
Reparación de hernia de Morgagni-Larrey con fijación al espesor total de la pared abdominal con puntos extracorpóreos. Reporte de un caso
}

\author{
Morgagni-Larrey hernia repair by full thickness anterior \\ abdominal wall fixation with extracorporeal knots: A case \\ report
}

José Eduardo Frías-Mantilla, José Asz-Sigall, María Antonieta Cabrera-Hernández

\begin{abstract}
Resumen
ANTECEDENTES: La hernia de Morgagni-Larrey es un padecimiento raro (3-5\% de todas las hernias diafragmáticas) estrechamente asociado con otras anomalías congénitas. La reparación de la hernia con afectación del grosor total de la pared abdominal anterior con puntos en " $U$ " extracorpóreos bajo visión directa fijados al tejido subcutáneo es una opción accesible para el cirujano experimentado pues permite máxima fuerza de reparación y adecuado control durante la colocación de los puntos de sutura. Esta técnica ha demostrado ser bien tolerada por el paciente con requerimientos mínimos de analgesia después de las primeras 24 horas, con buena recuperación y corta estancia intrahospitalaria.

CASO CLínICO: Paciente pediátrico intervenido con esta técnica en un hospital de tercer nivel.

PALABRAS CLAVE: Hernia diafragmática; hernia de Morgagni; cirugía de mínima invasión.

Abstract

BACKGROUND: The Morgagni-Larrey hernia (HML) represents a rare pathology, only $3 \%$ to $5 \%$ of all diaphragmatic hernias, being significantly associated with other congenital anomalies. The repair of the HML with the involvement of the total thickness of the anterior abdominal wall with extracorporeal $U$-shaped points under direct vision fixed to subcutaneous tissue represents an option accessible to the trained surgeon that allows maximum repair force and adequate control during the placement of stitches. This technique has also been shown to be well tolerated by the patient with minimum analgesia requirements after the first 24 hours with good recovery and short in-hospital stay. CLINICAL CASE: We present a patient operated with this technique in a specialty hospital. KEYWORDS: Pediatric Surgery; Diaphragmatic hernia; Morgagni hernia; Minimally invasive surgery.
\end{abstract}

${ }^{1}$ Servicio de Cirugía pediátrica.

Instituto Nacional de Pediatría, Ciudad de México.

Recibido: 24 de noviembre 2018

Aceptado: 16 de mayo 2019

Correspondencia

José Eduardo Frías Mantilla eduardofriasmantilla@gmail.com

Este artículo debe citarse como Frías-Mantilla JE, Asz-Sigall J, CabreraHernández MA. Reparación de hernia de Morgagni-Larrey con fijación al espesor total de la pared abdominal con puntos extracorpóreos. Reporte de un caso. Acta Pediatr Mex. 2109;40(4):211-16. 


\section{ANTECEDENTES}

La hernia de Morgagni-Larrey es un padecimiento raro (3 a 5\% de todas las hernias diafragmáticas) estrechamente asociado con otras anomalías congénitas. La hernia de Morgagni-Larrey se reporta en gemelos idénticos, lo que incrementa la posibilidad de una alteración hereditaria. Con el advenimiento de la cirugía de mínima invasión se han descrito diversos accesos laparoscópicos seguros, efectivos y con excelentes resultados cosméticos. Sin embargo, algunas de estas técnicas en niños requieren de maniobras laparoscópicas relativamente complejas. La reparación de la hernia de Morgagni-Larrey con afectación del grosor total de la pared abdominal anterior con puntos en " $U$ " extracorpóreos bajo visión directa fijados al tejido subcutáneo representa una opción accesible para el cirujano adiestrado, pues permite máxima fuerza de reparación y adecuado control durante la colocación de los puntos de sutura.

Esta técnica ha demostrado buena tolerancia por el paciente, con requerimientos mínimos de analgesia después de las primeras 24 horas, con buena recuperación y corta estancia intrahospitalaria.

\section{CASO CLÍNICO}

Niña de 1 año 4 meses, de $6.8 \mathrm{~kg}$ y $71 \mathrm{~cm}$; nacida de una madre de 43 años, con antecedente perinatal de amenaza de aborto durante el tercer mes del embarazo. La paciente cuenta con diagnóstico de base de síndrome de Down y antecedentes médicos de al menos 10 hospitalizaciones por infección de vías respiratorias, eventos de vómito y regurgitación. Al ingreso al INP se encontró asintomática, con saturación mayor de $90 \%$ al aire-ambiente, campos pulmonares con murmullo vesicular audible bilateral, precordio normodinámico, sin agregados, abdomen blando; el resto sin datos patológicos.

\section{Diagnóstico}

La radiografía de tórax (Figura 1) evidenció una imagen radiolúcida anterior, con silueta cardiaca de mayor extensión hacia el lado derecho del hemitórax. La tomografía de tórax confirmó el contenido abdominal en el tórax. Figura 2

Se solicitó valoración por cardiología con diagnóstico de forma en oval permeable sin repercusión hemodinámica, ni hipertensión arterial pulmonar. Los estudios de laboratorio prequirúrgicos fueron normales. Se programó para corrección quirúrgica.

\section{Descripción de la técnica quirúrgica}

Se colocó a la paciente en decúbito supino, con flexión y abducción de articulaciones coxofemorales y sujeción gentil en la mesa quirúrgica. Con técnica de Hasson se introdujo el trócar de visión $5 \mathrm{~mm}$, se indujo neumoperitoneo con presión de $10 \mathrm{~mm}$ de mercurio, flujo de $2 \mathrm{~L}$ por minuto y se realizó laparoscopia diagnóstica en la que

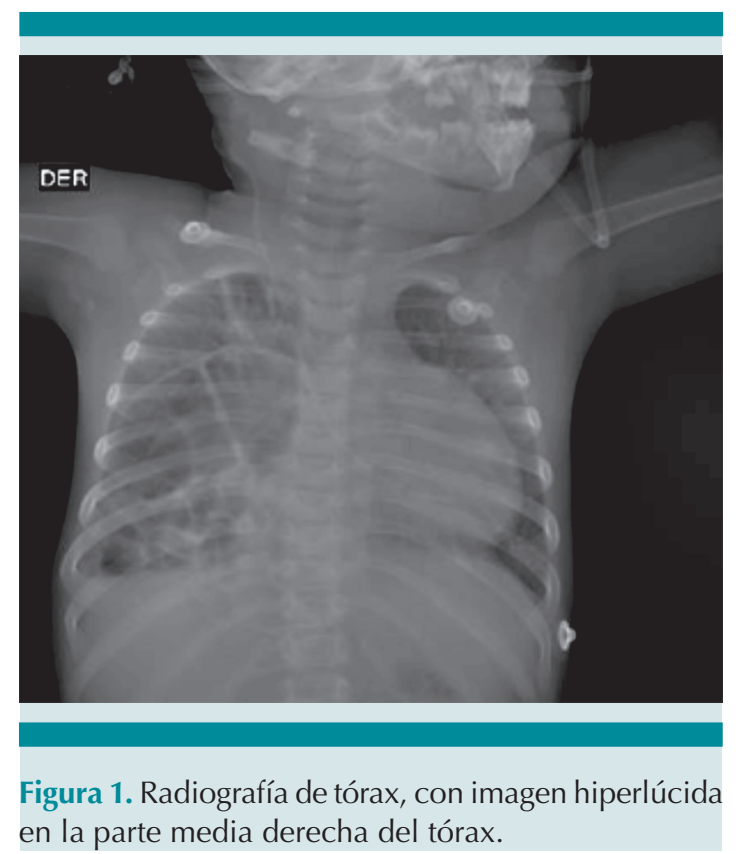




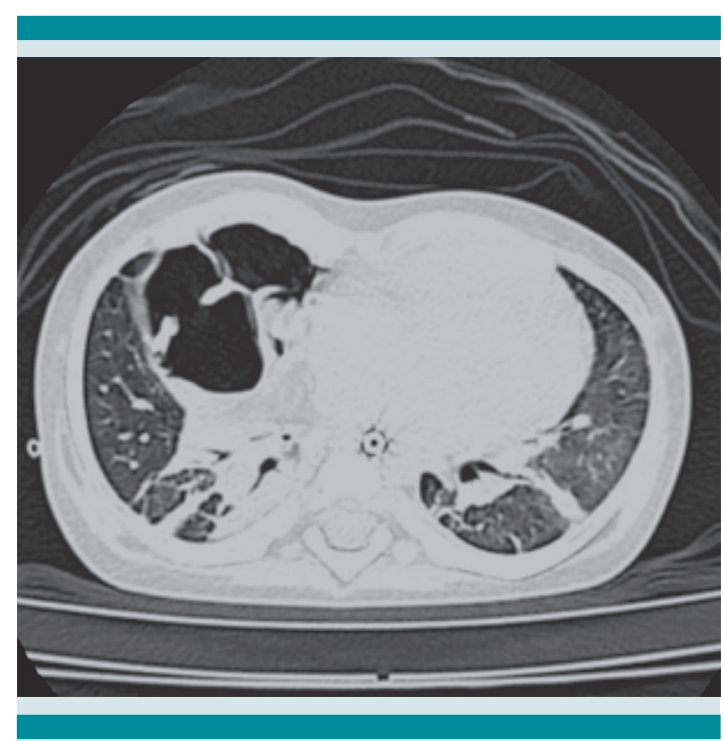

Figura 2. Tomografía que muestra un defecto diafragmático anterior. Desplazamiento del parénquima pulmonar hacia el lado derecho del hemitórax.

se encontró un defecto diafragmático derecho anterior, con colon ascendente y transverso en la cavidad torácica. Se colocaron dos trócares más de trabajo con técnica estándar y con visión directa, uno en la fosa iliaca derecha y otro subxifoideo, a $2 \mathrm{~cm}$ de la línea media.

Se procedió a retraer el contenido herniario, se expuso el saco, que se separó con disección; diatermia y roma de pleura, y pericardio en su totalidad. Una vez expuestos ambos labios del defecto diafragmático se colocaron puntos transcutáneos con prolene 3-0 de tejido subcutáneo al diafragma, pasando por ambos labios del defecto. Se comprobó el cierre completo del defecto y se anudó con puntos en el tejido celular subcutáneo. Figura 3

Se verificó la hemostasia. Al revisar la cavidad se encontró el ángulo de Treitz en el cuadrante superior izquierdo del abdomen, antes de la línea media, se descartó la mal rotación intestinal. Se retiraron los trócares de visión directa y neumo- peritoneo. Se procedió al cierre de incisiones de puertos, el umbilical con ácido poliglicólico de 3-0 aponeurosis y el resto de la piel con monofilamento absorbible de 4-0. Figura 4

\section{Seguimiento}

La paciente tuvo adecuada evolución posquirúrgica, sin suplementación de oxígeno desde el segundo día posquirúrgico, con tolerancia oral satisfactoria. Egresó al quinto día posoperatorio sin complicaciones. Durante el seguimiento en la consulta externa permaneció asintomática, sin requerir aporte de oxígeno. La última radiografía de tórax no mostró datos de recidiva.

\section{DISCUSIÓN}

La hernia de Morgagni-Larrey es un padecimiento raro (3-5\% de todas las hernias diafragmáticas), ${ }^{1,2}$ estrechamente relacionado con otras anomalías congénitas. La hernia de Morgagni-Larrey también se ha reportado en gemelos idénticos, lo que incrementa la posibilidad de una alteración hereditaria. $^{2}$

Desde la introducción de la cirugía de mínima invasión diversos accesos quirúrgicos laparoscópicos se han descrito como técnicas seguras y efectivas, con excelentes resultados cosméticos para el paciente. ${ }^{3,4}$ Sin embargo, algunas de estas técnicas en niños implican maniobras laparoscópicas relativamente complejas.

Larrey, un cirujano del ejército de Napoleón, fue el primero en describir el acceso abdominal retroesternal como una técnica sencilla para el drenaje pericárdico, al documentar que la hendidura antero-mediana y los triángulos esternocostales derecho (Morgagni) e izquierdo (Larrey) son sitios particularmente débiles del diafragma que permiten la sencilla comunicación entre ambas cavidades. La hernia de Morgagni-Larrey fue descrita por Giovanni Battista Morgagni en 1769. ${ }^{5}$ 

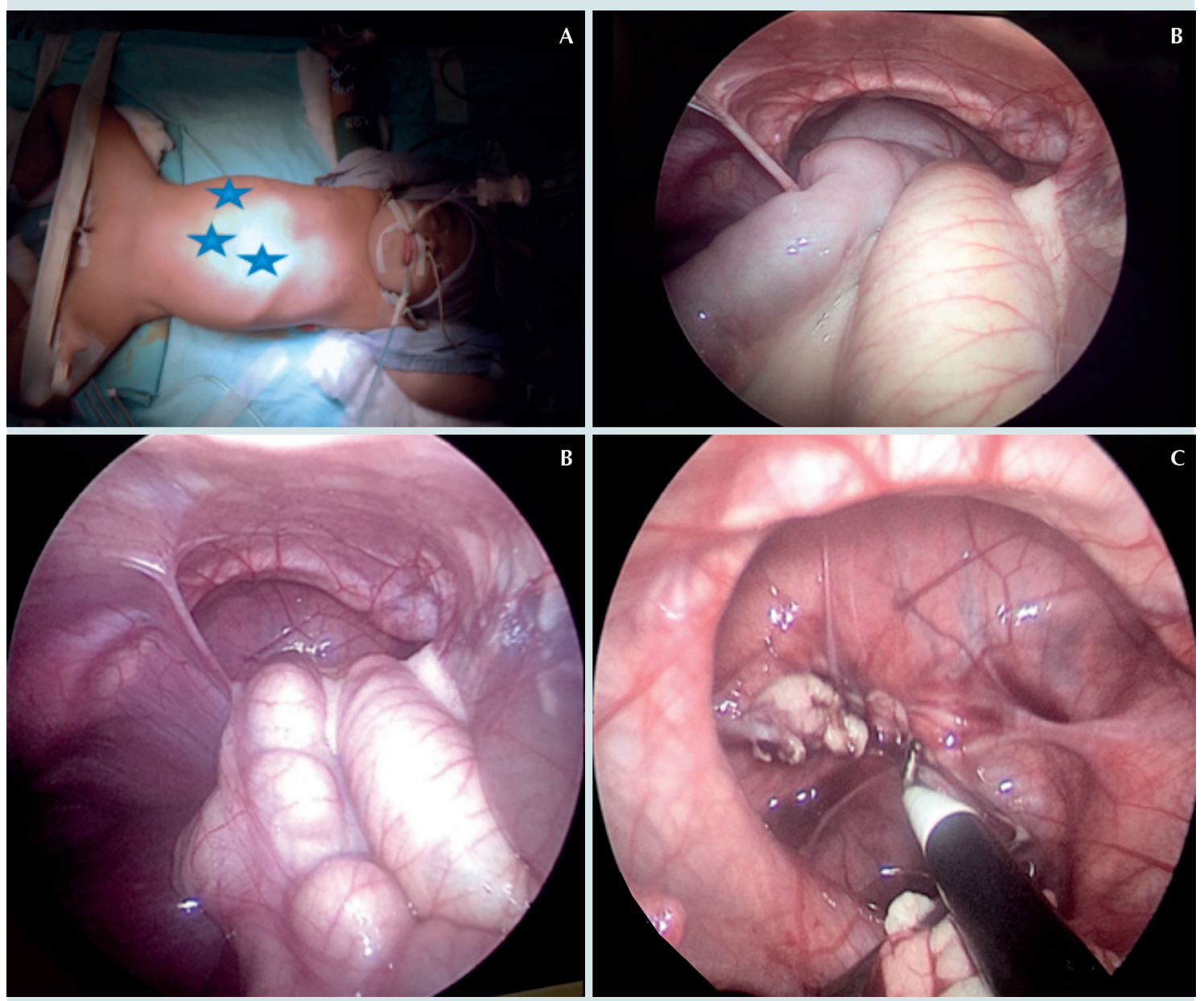

Figura 3. Posición de la paciente (A); resección del saco herniario (B, C Y D).

La primera reparación laparoscópica de hernia de Morgagni-Larrey fue reportada en 1992, en una mujer de 67 años, por Kuster y colaboradores; ${ }^{6}$ desde entonces diversas técnicas y abordajes han sido han sido descritos con resultados comparables a la cirugía abierta, con tasas de éxito cercanas a $100 \%, 2,7-10$ con bajas tasas de recurrencia, asociadas en pacientes con síndrome de Down, ${ }^{11}$ probablemente secundario a las características musculares hipotónicas de los pacientes, que pueden propiciar la aparición de herniaciones o dehiscencias. ${ }^{2,8}$

A partir de ese momento la cirugía de mínima invasión para hernia de Morgagni-Larrey fue rápidamente aceptada como protocolo de elección en la población infantil y adulta. ${ }^{12}$

Si bien la reducción del contenido abdominal es un proceso relativamente sencillo, es el cierre 

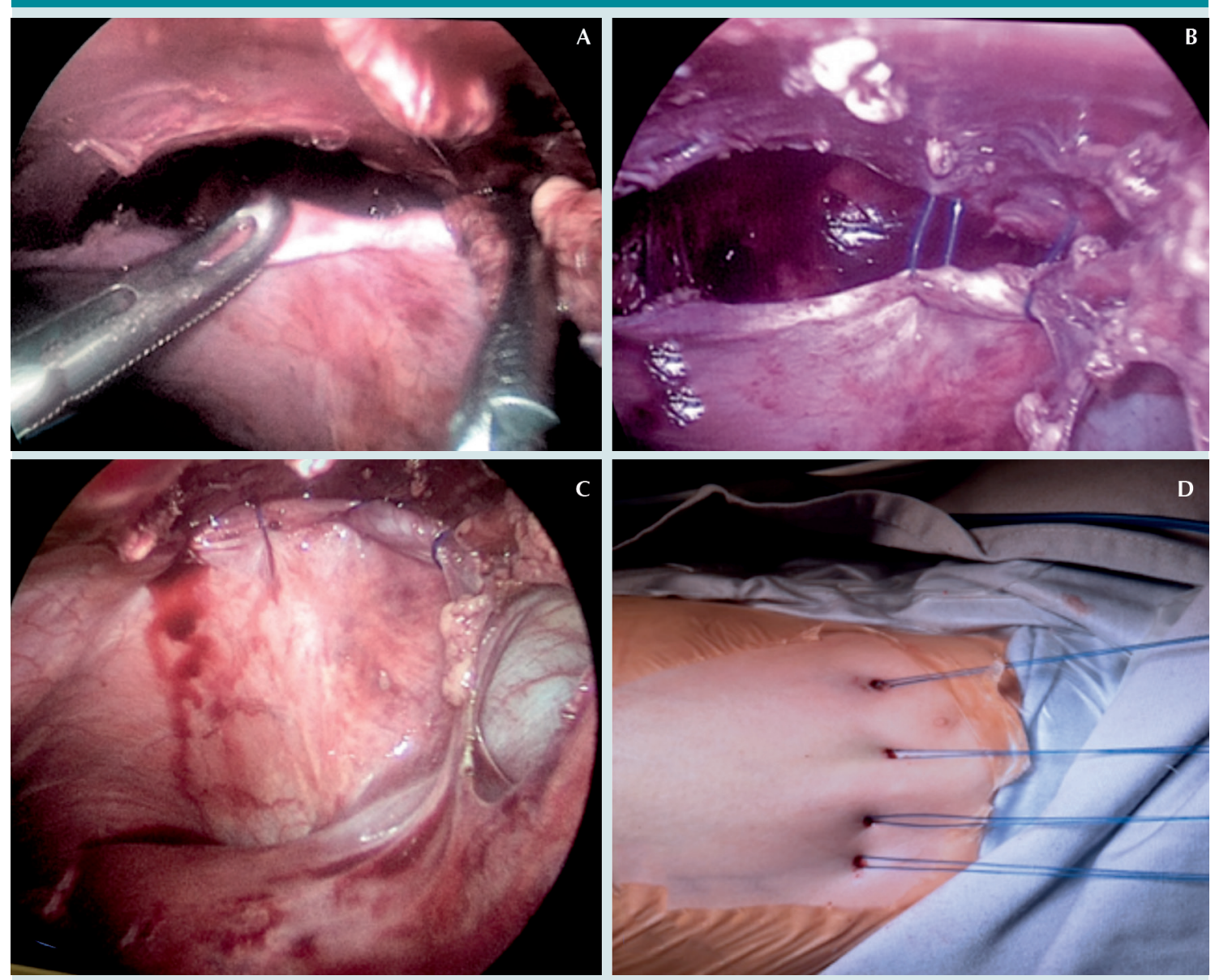

Figura 4. Defecto herniario (A); puntos de sutura en el espesor total de la pared (B y C) y vista final del procedimiento (D).

del defecto el que condiciona un reto quirúrgico, por efectuarse exclusivamente por medio de laparoscopia. Una de las técnicas más aceptadas es el anclaje de las suturas en el borde costal y en la cara posterior del esternón, técnica que resulta especialmente desafiante y donde la fascia relativamente débil puede representar una sujeción poco confiable.

Aunque poco descrita en cirugía pediátrica, la reparación laparoscópica de la hernia de Morgagni-Larrey con nudos extracorpóreos que incluyen el espesor total de la pared abdominal anterior bajo visión directa representa una sutura sólida que da máxima fuerza a la reparación, ${ }^{13}$ pues el diámetro transverso del defecto es significativamente mayor que el anteroposterior, lo que permite que este tipo de cierre mantenga fuerzas de tensión reducidas, fenómeno que facilita el cierre del mismo. ${ }^{5,12}$

Existen reportes de la resección del saco herniario y de intervenciones donde éste no es resecado con la plicatura local del saco, dejando esta maniobra a elección del cirujano. En el caso de nuestro procedimiento, este fue disecado con 
diatermia para facilitar el cierre del defecto y disminuir las consecuencias adversas que implican la plicatura del saco herniario; además, diversos autores indican que la resección del saco herniario disminuye la complejidad del procedimiento. ${ }^{8,9,14,15}$

En casos como el de nuestro paciente, donde el borde diafragmático anterior es prácticamente inexistente, la fijación al espesor total de la pared abdominal anterior está bien justificada como una excelente alternativa, que al realizarse con puntos en " $U$ " con fijación extracorpórea confiere adecuada fuerza a la fijación.

La técnica de fijación al grosor total de la pared abdominal anterior con nudos extracorpóreos es sencilla y segura pues ha demostrado a través de estudios su eficacia y la reducción de efectos adversos relacionados con otras técnicas laparoscópicas, por lo que representa una alternativa segura y viable en el paciente pediátrico.

\section{REFERENCIAS}

1. Tan $Y$, et al. Morgagni hernia repair in children over two decades: Institutional experience, systematic review, and meta-analysis of 296 patients. J Pediatr Surg. 2018;53(10):1883-1889.

2. Al-Salem A. Congenital hernia of Morgagni in infants and children. J Pediatr Surg. 2007;42(9):1539-1543.
3. Kashiwagi $\mathrm{H}$, et al. Morgagni hernia treated by reduced port surgery. International J Surg Case Rep. 2014;5(12):12221224.

4. Jaiswal S, et al. Congenital Morgagni hernia. Med J Armed Forces India. 2015;71(4):396-399.

5. Azzie G, et al. A simple technique of laparoscopic fullthickness anterior abdominal wall repair of retrosternal (Morgagni) hernias. J Pediatr Surg. 2003;38(5):768-770.

6. Becmeur F, et al. Laparoscopic surgery of Morgagni-Larrey hernias: A Multicenter Study of the Groupe d'Étudeen Coeliochirurgie Infantile (GECI). Pediatr Endosurg Innov Tech 2003;7(2):147-152.

7. Kuster G, et al. Diaphragmatic hernia through the foramen of Morgagni: laparoscopic repair Case Report. J Laparoendosc Surg 1992;2(2):93-100

8. Escarcega $\mathrm{P}$, et al. Multi-institution case series of pediatric patients with laparoscopic repair of Morgagni hernia. J Laparoendosc Adv Surg Tech A 2018;28(8):1019-1022. doi: 10.1089/lap.2017.0621.

9. Golden J, et al. Pediatric Morgagni diaphragmatic hernia: a descriptive study. Pediatr Surg Int 2017;33(7):771-775.

10. Papia G, et al. Laparoscopic repair of Morgagni diaphragmatic hernia in children: Technical challenges and results. Pediatr Endosurg Innov Tech 2004;8(3):245-249.

11. Cigdem $\mathrm{M}$, et al. Associated malformations in Morgagni hernia. Pediatr Surg Int 2007;23(11):1101-1103.

12. Lamas-Pinheiro $\mathrm{R}$, et al. Minimally invasive repair of Morgagni hernia-A multicenter case series. Rev Port Pneumol (English Edition). 2016;22(5):273-278.

13. Mallick M, et al. Laparoscopic-assisted repair of Morgagni hernia in children. J Pediatr Surg 2009;44(8):1621-1624.

14. Garriboli M, et al. Recurrence rate of Morgagni diaphragmatic hernia following laparoscopic repair. Pediatr Surg Int. 2012; 29(2):185-189.

15. Esposito $\mathrm{C}$, et al. Technical standardization of laparoscopic repair of Morgagni diaphragmatic hernia in children: results of a multicentric survey on 43 patients. Surg Endosc 2016;31(8):3320-3325. 